\title{
DIRECTOR(A) POR PRIMERA VEZ. UN ESTUDIO SOBRE LA EXPERIENCIA Y SOCIALIZACIÓN DE LOS DIRECTORES NOVELES EN ESTABLECIMIENTOS MUNICIPALES DE CHILE ${ }^{1}$
}

\author{
José Weinstein ${ }^{2}$, Carolina Cuéllar ${ }^{3}$, Macarena Hernández ${ }^{4}$, \\ Magdalena Fernández $z^{5}$
}

\section{RESUMEN}

El ejercicio por primera vez de la posición de director(a) de un establecimiento escolar es un período crítico para el desempeño del cargo que no ha sido prácticamente estudiado en Chile, a pesar de la amplia renovación de directivos escolares en curso. El presente artículo indaga, sobre la base de 12 estudios de caso y una encuesta aplicada a 76 directores, en la experiencia y la socialización de los directores novatos del sector municipal, describiendo de paso cuáles son sus principales necesidades formativas. Los resultados más importantes son: la elaboración de un perfil de los directores principiantes; la constatación de su abrupta llegada al establecimiento; la priorización de actividades centradas en el corto plazo, con énfasis en la convivencia y la gestión institucional (relegando a la gestión pedagógica); la experimentación de dificultades externas e internas al establecimiento que se combinan con la carencia de competencias personales para enfrentarlas; la ausencia de apoyos institucionalizados para este período inicial, lo que es parcialmente suplido por actores internos y por la propia red personal de directores cercanos; y la necesidad de distinguir la experiencia vivida por los directores noveles según su carácter de insider u outsider al establecimiento, así como si provienen o no del sector municipal.

Palabras clave: directores noveles, Chile, liderazgo escolar

\section{FIRST TIME PRINCIPAL. A STUDY ABOUT THE EXPERIENCE AND SOCIALIZATION OF NEW PUBLIC SCHOOL PRINCIPALS IN CHILE}

\begin{abstract}
Taking the role of a school principal for the first time is a critical period in their job performance, issue that has been sparsely studied in Chile despite of the increasing renovation of school leaders at the time. Based on 12 case studies and a survey administered to 76 principals, this article explores the experience and socialization of beginning principals at public schools, capturing their professional development needs as well. The main results include: a profile of beginning principals; the confirmation of an abrupt start at school organization; focus on short term actions emphasizing school climate and institutional management actions (delaying focus on pedagogical management); experiencing external and internal school difficulties combined with a lack of personal competencies to overcome challenges; the absence of institutional support during this initial period, which is compensated by school internal actors' support and individuals' personal networks with other principals; and the need to differentiate between the experiences of beginning principals depending on whether they are insiders or outsiders to the school organization or the municipal sector.
\end{abstract}

Keywords: Beginning principals, Chile, school leadership

1 Este artículo se basa en los resultados obtenidos en el desarrollo del proyecto Fonide n ${ }^{\circ}$ F911419 "El primer año de los directores novatos. Orientaciones para un programa de inducción profesional".

2 Facultad de Educación, Universidad Diego Portales, Santiago, Chile. Contacto: jose.weinstein@udp.cl

3 Doctorado en Educación de la Universidad Diego Portales y la Universidad Alberto Hurtado, Santiago, Chile.

4 Doctorado en Educación de la Pontificia Universidad Católica de Chile, Santiago, Chile.

5 Doctorado en Educación, Universidad de Toronto, Toronto, Canadá. 
"Fui como aprendiendo sobre la marcha lo que significaba, más en la experiencia..." (director novato)

"Estoy como con demasiadas cosas y no he podido organizar, no puedo organizar mis tiempos. Yo creo que eso es lo que más me cuesta" (directora novata)

\section{Introducción}

La política educativa chilena está enfrentada a una situación desafiante: en un contexto de alta responsabilización de los directivos ${ }^{6}$ por los resultados escolares de los establecimientos (Weinstein y Muñoz, 2012; Weinstein, Muñoz \& Raczynski, 2011), está en marcha una amplia renovación del contingente de directores en escuelas y liceos públicos. Según mandata la Ley No 20.501, a fines del año 2016 todos los directores de establecimientos escolares del sector municipal deberán haber sido nombrados bajo la nueva modalidad vigente, con participación del Servicio Civil y de la Alta Administración Pública; razón por la cual en el futuro inmediato deberán seguirse produciendo numerosos concursos y nombramientos de directivos escolares (Grupo Educativo, 2015).

Por ello es importante hacer frente a la situación que viven los directores noveles o principiantes -aquellos que por primera vez ocupan la posición directiva- quienes, como ha sido retratado por la literatura especializada (Blase, 1985; Daresh, 1987; Daresh \& Male, 2000; Earley et al., 2011; García Garduño, Slater \& LópezGorosave, 2011a; Greenfield, 1985; Leithwood, Steinbach \& Begley, 1992; Oplatka, 2012; Parkay \& Hall, 1992; Spillane \& Lee, 2013; Walker \& Qian, 2006), se enfrentan a grandes y complejas tensiones en su nuevo cargo y requieren de estrategias específicas de apoyo (Hargreaves, Moore, Fink, Brayman \& White, 2003). De hecho, el desafío de proveer de un acompañamiento a los directores novatos se encuentra establecido en la hoja de ruta del actual gobierno, que

6 Con el fin de facilitar la lectura y respetar el principio lingüístico de la economía expresiva, se utilizará durante el texto el masculino (director, directores) siguiendo el precepto de que, según la Real Academia de la Lengua Española, se puede usar como representante genérico inclusivo de hombres y mujeres. 
14 DIRECTOR(A) POR PRIMERA VEZ. UN ESTUDIO SOBRE LA EXPERIENCIA Y SOCIALIZACIÓN DE LOS DIRECTORES NOVELES EN ESTABLECIMIENTOS MUNICIPALES DE CHILE - J. Weinstein, C. Cuéllar, M. Hernández y M. Fernández

anuncia la creación de un programa de inducción para directivos escolares. Sin embargo, dicho programa -o cualquier otra iniciativa similar-solo logrará ser efectivo si se basa en un real conocimiento de los desafíos profesionales y personales que los directores noveles deben enfrentar en el particular contexto escolar del país -tema sobre el cual existe un pronunciado vacío de conocimiento empírico(Weinstein y Muñoz, 2012).

Más allá de nuestras fronteras, la temática de los directores principiantes ha cobrado relieve a nivel internacional, en especial en torno al estudio del liderazgo y la mejora escolar (García Garduño, Slater \& López-Gorosave, 2011b). Los primeros estudios acerca del tema se publicaron en Estados Unidos a fines de 1970 (Greenfield 1977), siendo seguidos por investigaciones pioneras en Inglaterra (Daresh, 1987; Weindling y Earley, 1987;) y en otros países de origen anglosajón. En la década de 2000, la temática comenzó a proliferar y a convertirse en un campo de estudio especializado. Hoy en día, esta línea se ha fortalecido con la emergencia de redes de investigación internacionales, que cubren países anglosajones y no anglosajones, como es el International Beginning Principal Study (IBPS) del que participan países como Estados Unidos, Australia, Bélgica, Reino Unido, Holanda, Tailandia, Taiwán y Corea; y el International Study Principal Preparation (ISPP) que incluye a Australia, Canadá, China, Inglaterra, Alemania, Jamaica, Kenia, México, Nueva Zelandia, Escocia, Sudáfrica, Tanzania, Turquía y Estados Unidos (García Garduño et. al, 2011b).

Este artículo, basado en un estudio recientemente concluido por los autores, pretende justamente profundizar en lo que les acontece a los directores novatos que están ingresando a los establecimientos públicos. Más precisamente, las preguntas que lo orientan son las siguientes:

- ¿Cómo es la experiencia de desempeño en el cargo de los directores novatos en el primer año de su gestión?

- ¿Cómo son los procesos de socialización de los directores novatos al cargo? 
- ¿Cuáles son las principales necesidades formativas de los directores novatos?

El artículo parte por exponer los conceptos centrales que guían la investigación, luego presenta la metodología empleada, posteriormente sintetiza los principales hallazgos realizados y termina recapitulando los resultados principales y abriendo ciertas discusiones que aquellos concitan.

\section{Dos conceptos clave: experiencia y socialización}

La mayor parte de los estudios enfocados en la transición al cargo de los directores novatos ha respondido al objetivo central de caracterizar la experiencia de desempeño en el rol durante el primer o primeros años de la gestión. Dentro del concepto general de experiencia, se han estudiado -aunque con más o menos relevancia y profundidadtres temas focales:

- la adecuación o no adecuación entre las expectativas y la realidad del cargo;

- los desafíos y dificultades centrales encontrados; y

- las tareas y acciones iniciales emprendidas por los directivos en el período.

Un consenso generalizado dentro la literatura es que la transición al cargo directivo es experimentada como un shock de realidad e incluso como una experiencia traumática (Daresh \& Male, 2000; García Garduño et al., 2011a; Spilane \& Lee, 2013). Así, un aspecto que marca la experiencia de los directores novatos es la sorpresa y escasa anticipación que poseen respecto de la complejidad del rol, así como las múltiples exigencias y demandas que provienen desde actores internos y externos al establecimiento, a las que deben responder siendo, por primera vez, los responsables últimos de lo que ocurra dentro de la organización (Crow, 2007; Daresh \& Male, 2000; Kelly \& Saunders, 2010; Oplatka, 2012; Spillane \& Lee, 2013).

Buscando desentrañar los elementos centrales de este shock de realidad, una amplia gama de investigaciones se ha abocado a 
16 DIRECTOR(A) POR PRIMERA VEZ. UN ESTUDIO SOBRE LA EXPERIENCIA Y SOCIALIZACIÓN DE LOS DIRECTORES NOVELES EN ESTABLECIMIENTOS MUNICIPALES DE CHILE - J. Weinstein, C. Cuéllar, M. Hernández y M. Fernández

profundizar, desde metodologías cualitativas y particularmente estudios de caso, los principales desafíos de los directivos durante su primer año. Así, la revisión de estudios realizados a lo largo de la última década muestra que dentro de las problemáticas más recurrentes están: la soledad, aislamiento o abandono percibido; el lidiar con la cultura escolar establecida y el legado del director o la directora anterior; el manejo y relaciones con el staff; el manejo del tiempo y las prioridades en la gestión; y el volumen, diversidad e impredictibilidad de las tareas asociadas al cargo (Crow, 2007; Earley et al., 2011; García Garduño et al., 2011b; Spillane \& Lee, 2013; Walker \& Qian, 2006). Este conjunto de tensiones y dificultades es un hecho reiterado, como lo muestran los estudios comparados, en diferentes países y sistemas escolares (Nelson \& Slater, 2013). En la investigación chilena relacionada con directores escolares en escuelas municipales, aunque no referida a los directores novatos en particular, existe evidencia acerca de las dificultades similares enfrentadas por los líderes, las que coinciden en problemáticas como la organización y utilización del tiempo, y la alta dedicación a tareas ajenas a la gestión curricular. Paralelamente, presentan otros problemas como la ausencia de atribuciones para la administración de recursos financieros, carencia de recursos materiales, o la ausencia de facultades y atribuciones para la selección y despido del personal (Ulloa, Nail, Castro y Muñoz, 2012; Weinstein y Muñoz, 2012).

La literatura ha avanzado hacia la determinación de las distintas acciones y tareas que emprenden los líderes educativos al ascender al cargo e iniciar su labor en una organización escolar específica. Parte fundamental de los marcos de referencia relativos a este tema se han basado en la literatura organizacional más amplia que ha identificado las etapas que experimentan los líderes en su llegada a un cargo de mayor jerarquía.

Así, durante el primer año, las principales tareas desarrolladas por los líderes corresponden al entendimiento de la estructura y cultura organizacional, la fijación de prioridades, la respuesta a los cambios más urgentes y la evaluación del personal. El estudio desarrollado por Parkay y Hall (1992) establece que las principales acciones que toman los directivos durante sus primeros meses en el cargo corresponden a 
la familiarización con la escuela, dentro de la cual se incluyen aspectos variados tales como la obtención de información sobre resultados académicos, la infraestructura y las políticas; el establecimiento de prioridades y de una planificación anual; el desarrollo del staff y de la cultura escolar; y la normalización de la escuela (p.e., establecimiento de reglas de disciplina y trabajo escolar). Estudios más recientes han generado evidencia similar (Earley et al., 2011) y han enfatizado que las primeras operaciones de los directores en las escuelas se desarrollan "hacia adentro" antes que "hacia afuera", e incluyen fundamentalmente el asegurar la aceptación del personal, la construcción de alianzas de apoyo internas y la distribución del liderazgo, antes de avanzar hacia la toma de decisiones estratégicas y los asuntos externos (Kelly $\&$ Saunders, 2010).

En Chile, la evidencia respecto de las acciones emprendidas por directores escolares en general, menciona también que estos se orientan hacia el desarrollo de condiciones internas en la organización (p. ej., modificación de relaciones, creencias, acceso a la información, desarrollo técnico, o la definición de roles y toma de decisiones), centradas en el "hacer actual", sin prestar atención a dimensiones estratégicas como la construcción de capacidades (Rodríguez, Cárdenas y Campos, 2012).

En paralelo, distintos estudios han abordado la socialización al cargo, es decir, el proceso bajo el cual un individuo adquiere en forma selectiva los conocimientos, habilidades y disposiciones para desempeñar adecuadamente un rol social dentro de un grupo en el que buscan ser o son miembros (Blase, 1985; Greenfield, 1985; Lacey, 1977; Merton 1957, 1963, 1968 citado en Leithwood et al., 1992; Parkway \& Hall, 1992; Van Maanen \& Schein, 1979; Wentworth, 1980). Con el fin de integrar la conceptualización de la socialización procedente de la sociología tradicional-que la define como un proceso en el que el individuo internaliza aspectos de la sociedad de forma pasiva (Lacey, 1977)- con las críticas desde la tradición interpretativa de la misma disciplina -que argumenta que la socialización ocurre como resultado de la intersubjetividad que emerge en la inmediata interacción entre individuos (Wentworth, 1980)-, las corrientes teóricas actuales han complementado ambos polos interpretativos, 
situando a la socialización en un lugar intermedio, en el que el individuo es ciertamente influenciado por la sociedad, pero al mismo tiempo internaliza selectivamente los conocimientos, habilidades y disposiciones necesarias para desempeñar un rol social. Desde esta visión comprensiva del proceso, la teoría de la socialización permite incluir aspectos ligados con el contexto institucional y con la propia biografía personal, como elementos que interactúan en el proceso de aprendizaje de los individuos (Blase, 1985, 1986).

Dentro del proceso de socialización, la literatura enfocada en la transición al cargo de los directores noveles ha situado su atención en tres elementos clave:

- los tipos de socialización y sus fuentes predominantes;

- las características de los procesos (o tácticas) y de los métodos de socialización; y

- los contenidos de la socialización.

El Cuadro 1 sintetiza dichos contenidos: 
Cuadro 1. Resumen de conceptos referidos a la socialización de los directores novatos

\begin{tabular}{|c|c|c|}
\hline Tipos de socialización & Procesos, métodos y fuentes & Contenidos \\
\hline $\begin{array}{l}\text { Anticipatoria } \\
\text {-Antes de asumir el cargo en forma } \\
\text { consciente o inconsciente. } \\
\text { - Potencialmente desde que fue } \\
\text { estudiante, preparación formal como } \\
\text { profesor, mientras fue profesor de } \\
\text { aula, y en la ocupación de otro rol de } \\
\text { liderazgo en la escuela. } \\
\text {-Destaca el rol de mentores. }\end{array}$ & $\begin{array}{l}\text { Procesos } \\
\text { 1. Colectivo vs. individual } \\
\text { 2. Formales vs. informales } \\
\text { 3. Secuencial vs. aleatorias } \\
\text { 4. Fijas vs. variables } \\
\text { 5. Seriales vs. disyuntivas } \\
\text { 6. Investidura vs. despojo } \\
\text { Métodos }\end{array}$ & $\begin{array}{l}\text { Técnicos } \\
\text { Conocimientos } \\
\text { Habilidades } \\
\text { Morales } \\
\text { Normas y valores en el } \\
\text { contexto escolar } \\
\text { Contextuales }\end{array}$ \\
\hline $\begin{array}{l}\text { Profesional } \\
\text {-Experiencias comunes a todos los } \\
\text { directores antes de ejercer. } \\
\text {-Pueden darse en el proceso de } \\
\text { selección, formación o certificación } \\
\text { preparatoria al cargo u otra } \\
\text { instancia formal o informal durante } \\
\text { el ejercicio del cargo, pero común a } \\
\text { todos los profesionales. }\end{array}$ & $\begin{array}{l}\text { 1. Mentoría y coaching } \\
\text { 2. Aprendizaje virtual y uso de ICT } \\
\text { 3. Trabajo en redes } \\
\text { 4. Proyectos basados en escuelas } \\
\text { Fuentes (individuos) } \\
\text { 1. Profesores } \\
\text { 2. Superiores o autoridades } \\
\text { locales }\end{array}$ & $\begin{array}{l}\text { Conocimientos sobre: } \\
\text { Contexto } \\
\text { Personas }\end{array}$ \\
\hline $\begin{array}{l}\text { Organizacional } \\
\text {-En el trabajo, en el contexto } \\
\text { específico de la escuela en que se } \\
\text { desempeña por primera vez como } \\
\text { director. } \\
\text {-Describen instancias de interacción } \\
\text { con otros actores internos y } \\
\text { externos a la escuela como las más } \\
\text { relevantes. }\end{array}$ & $\begin{array}{l}\text { 3. Miembros del equipo directivo } \\
\text { 4. Personal del colegio } \\
\text { 5. Asesores técnicos externos } \\
\text { 6. Padres y apoderados } \\
\text { 7. Estudiantes } \\
\text { 8. Pares, directores en otras } \\
\text { escuelas } \\
\text { 9. Otros externos a comunidad } \\
\text { escolar } \\
\text { 10. Familiares del director }\end{array}$ & Sistema \\
\hline
\end{tabular}

Fuente: Elaboración propia a partir de Crow (2007), Van Maanen y Schein (1979), Daresh y Male (2000), Duke (1987), Weinstein y Hernández (2015b), Clarke y Wildy (2013), Greenfield (1985)

En la investigación acerca del liderazgo escolar en general, nuevas tendencias han reclamado por una mayor consideración del contexto sociocultural y de políticas de los distintos sistemas escolares (Bush 2012; Hallinger \& Huber 2012; Weinstein \& Hernández, 2015a), debido a su repercusión en las prácticas y los procesos organizativos (Ahumada, 2012). En esta línea, la temática específica de los directivos noveles debiera abordarse dentro de sus particulares contextos organizacionales, societales, culturales, éticos, de género y estructurales (Walker \& Qian, 2006). De hecho, diversos estudios realizados en países no anglosajones han dado cuenta de desafíos adicionales enfrentados por los directivos principiantes, que pueden asociarse al contexto sociocultural y político mayor en el que se insertan. La evidencia en países como China, Corea, Tailandia, Sudáfrica o 
México, sistematizada por García Garduño y otros autores (2011b), permite afirmar que, aun cuando hay problemáticas similares a las de los países anglosajones, existen también particularidades, tales como:

- la presión por la implementación de las reformas educativas;

- la alta carga de trabajo administrativo;

- el ausentismo y desmotivación de muchos profesores; y

- la falta de recursos económicos de numerosas familias y estudiantes.

\section{Diseño de la investigación}

En el estudio realizado se definió un diseño metodológico secuencial mixto (Teddlie \& Tashakkori, 2006), que combina, en tres fases sucesivas, técnicas de recolección de información cualitativas y cuantitativas, con el doble propósito de comprender la experiencia y socialización de los directores noveles en profundidad y contar con resultados generalizables para la dependencia municipal. Dado el vacío de información existente en Chile, se optó por iniciar el diseño con una fase cualitativa que sirviera como insumo para la etapa cuantitativa, siendo, como puede apreciarse en el Cuadro 2, ambas trianguladas en la etapa final.

Cuadro 2. Diseño metodológico general del estudio

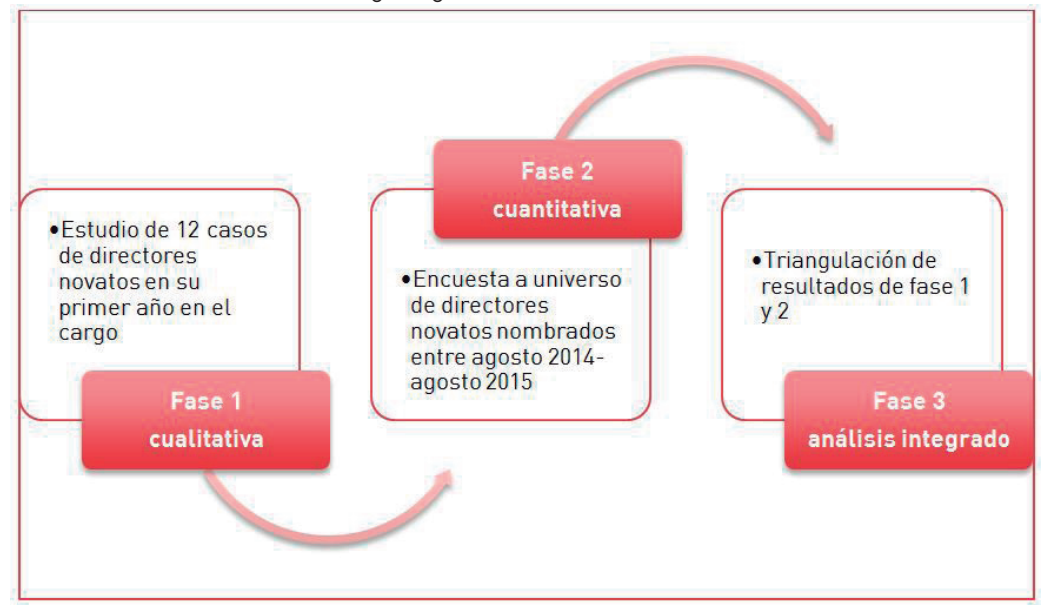

Fuente: Elaboración propia 
El estudio de los casos permitió investigar la experiencia y socialización de los directores principiantes en su contexto, posibilitando ahondar de manera comprehensiva en las unidades de análisis consideradas (Earley, 2012; Yin, 1999). Se seleccionaron 12 casos con el objetivo de dar cuenta de diferentes situaciones entre los novatos y que, de acuerdo con la literatura (Daresh \& Male, 2000; Day, Hopkins, Harris \& Ahtaridou, 2009; Hobson et al., 2003; Parkay $\&$ Hall, 1992; Spillane \& Lee, 2013; Weindling, 2000) son relevantes para el problema en estudio: el género (hombre/mujer); si ya trabajaba (insider) o no (outsider) en el establecimiento educativo donde ha sido nombrado; y el nivel de enseñanza (básica/media) y modalidad de enseñanza (media HC/media TP) del centro escolar que lidera ${ }^{7}$.

Los casos se seleccionaron en tres regiones del país (Metropolitana, Libertador Bernardo O’Higgins y del Maule) donde se habían producido nombramientos en el período en estudio. Cada caso se estudió en dos momentos del año escolar, aplicándose una batería variada de técnicas de recolección de información: entrevistas en profundidad y shadowing al director; entrevistas grupales a docentes, directivos, apoderados y al sostenedor; y revisión de documentos institucionales. La aplicación del shadowing (Early, 2012; McDonald, 2005) se realizó en dos jornadas laborales consecutivas durante la primera etapa del estudio, de manera de obtener una visión profunda, enmarcada en el contexto de trabajo, de la experiencia cotidiana de los directores, incluyendo el seguimiento a las diversas actividades realizadas por los actores y sus relaciones con otros miembros de la comunidad escolar y externos a esta. Las entrevistas a los distintos actores se llevaron a cabo en ambos momentos de estudio, siguiendo pautas de entrevistas semiestructuradas (LeCompte \& Preissle, 1993). Finalmente, la revisión de documentos consideró aquellos asociados con el proceso de selección de cada director y su convenio de desempeño. La información emanada de las distintas técnicas se estudió siguiendo las distintas etapas del análisis de contenido (Cohen, Manion \& Morrison, 2011), con el apoyo de una matriz de

7 El análisis referido a las implicancias acerca de la experiencia del principiante del género y del nivel de enseñanza del establecimiento que encabeza no han sido mayormente desarrollado en este artículo por problemas de espacio. 
vaciado para cada caso que incluyó las distintas dimensiones bajo investigación. Posteriormente, cada caso fue sistematizado por el respectivo investigador(a) responsable sobre la base de un índice estructurado, siendo todos estos informes sometidos a un análisis transversal. Así, la fase cualitativa del estudio siguió las estrategias de validación descritas por Shenton (2004), tales como la adopción de métodos de investigación establecidos, la triangulación de diversos informantes y la revisión y reflexión acerca de los casos entre los investigadores del equipo.

La segunda fase consistió en el diseño y aplicación de una encuesta al universo de directores novatos de establecimientos municipales nombrados entre agosto 2014 y agosto 2015. El cuestionario aplicado se construyó sobre la base de los hallazgos de los 12 casos, agregando algunas preguntas pertinentes provenientes de otros estudios internacionales. El instrumento elaborado fue piloteado con exdirectores de escuela, de manera de asegurar su validez (Cohen et al., 2011). De los 106 directores principiantes nombrados en el período, se logró una tasa de respuesta completa de 75\% (con $4,84 \%$ de error muestral al 95\% de confianza) y de 79\% (con 3,99\% de error muestral), si se añaden las cinco encuestas completadas parcialmente que fueron utilizadas en el análisis. La encuesta se aplicó con una plataforma online especialmente configurada en el programa SurveyMonkey y posteriormente se procesó la información, para un análisis descriptivo, utilizando el programa SPSS.

Finalmente se procedió a la triangulación metodológica de la información cualitativa y cuantitativa relevada (Cohen et al., 2011), utilizando las categorías conceptuales desarrolladas previamente.

\section{Principales resultados del estudio}

Es posible sintetizar los resultados obtenidos sobre los directores novatos en torno a cuatro temas mayores: su perfil y motivaciones; su llegada al establecimiento; las actividades desarrolladas, logros percibidos y dificultades encontradas; y la preparación previa, los apoyos reales y los apoyos deseados. 


\section{a. Perfil diferenciado y motivación por la tarea pública}

Dos rasgos sobresalientes del perfil de los principiantes en relación con el perfil de los directores municipales en servicio fueron: la mayor feminización del rol y el aumento cualitativo en la formación especializada para el desempeño de este. En efecto, los directores novatos que ingresaron a la posición durante el período estudiado son (casi) dos tercios mujeres, mientras que en el conjunto de los directores del sector municipal hay una proporción equivalente por género -con incluso un leve predominio masculino- (Mineduc, 2015). Igualmente, si bien los principiantes comparten con el conjunto de los directivos municipales la masiva asistencia a actividades de formación para el desempeño del cargo (UDP, 2014), la alta tenencia de magísteres, con casi cuatro de cada cinco habiendo alcanzado dicho grado, es otro rasgo que les caracteriza, por sobre el conjunto de directivos que han realizado en mayor medida diplomados o cursos de perfeccionamiento.

Un aspecto en que los directores noveles no muestran homogeneidad es en su experiencia laboral y edad, no siendo sinónimo el ser joven (en términos profesionales y biológicos) con la asunción del cargo. Así el promedio de experiencia previa es de 20 años, pero existe una amplia variabilidad (que oscila entre 6 y 46 años con una desviación estándar de 10 años). De manera que hay una proporción significativa (34\%) de principiantes experimentados que tienen entre 14 y 22 años de experiencia laboral, y una cifra similar que cuenta con 23 años o más de trayectoria anterior en escuelas y liceos.

Dentro de esta experiencia previa, un rasgo universal entre los novatos es el haber sido docente de aula. De la misma forma, una aplastante mayoría ha desempeñado algún cargo directivo, siendo los más frecuentes el de jefe de unidad técnico-pedagógica (55\%), jefe de departamento, ciclo o nivel (46\%), e inspector (24\%). De hecho, un $80 \%$ de los encuestados se desempeñaba en un cargo directivo inmediatamente antes de asumir como director y solo uno de cada cinco se movilizó directamente desde el aula hasta la dirección, lo que muestra una fuerte tendencia hacia la progresión paulatina y gradual. Es en el desempeño previo de estos diferentes cargos de 
24 DIRECTOR(A) POR PRIMERA VEZ. UN ESTUDIO SOBRE LA EXPERIENCIA Y SOCIALIZACIÓN DE LOS DIRECTORES NOVELES EN ESTABLECIMIENTOS MUNICIPALES DE CHILE - J. Weinstein, C. Cuéllar, M. Hernández y M. Fernández

jefatura cuando estos directores tomaron conciencia de que disponían de competencias, habilidades y motivación para ejercer la dirección escolar.

Otro elemento de interés, y que da cuenta de la fluidez laboral actualmente existente al interior del sistema escolar chileno, es la alta proporción de directores novatos que proviene del sector privado o bien que ha trabajado en él en algún período profesional pasado. Del conjunto de principiantes, solo hay un minoritario $27 \%$ que ha trabajado exclusivamente en el sector municipal, mientras que un 24\% solo lo ha hecho previamente en establecimientos particulares. Un 49\% ha trabajado en distintas dependencias.

En cuanto al conocimiento previo que los novatos tenían de su actual establecimiento, solo uno de cada cinco trabajaba en el mismo establecimiento escolar del que ahora es director (insider). Un grupo equivalente (23\%) proviene de otro establecimiento público dentro de la misma comuna. El grupo de directivos que procede de instituciones más distantes, sea geográfica o institucionalmente, no es menor: 25\% llegó al cargo desde un establecimiento particular (subvencionado o privado) y un $17 \%$ desde otro tipo de institución educativa (Daem, Mineduc, etc.).

Desde el punto de vista de las motivaciones declaradas por los directores noveles, destaca el interés por hacer un aporte a la educación pública y a la educación de los alumnos más desaventajados. Este deseo de realizar una acción con orientación pública es particularmente marcado entre quienes provienen del sector privado. Entre las motivaciones más orientadas hacia la propia carrera personal destaca el deseo de plantearse nuevos desafíos profesionales por sobre los vinculados a los eventuales incentivos propios de la posición, tales como la remuneración o la estabilidad. La Tabla 1 muestra dicha distribución de las motivaciones. 
Tabla 1. Principal motivación para postular al cargo en un establecimiento municipal $(\%)(\mathrm{N}=83)$

\begin{tabular}{l|c}
\hline & $\%$ \\
\hline Realizar una contribución a la gestión de la educación pública municipal & 55 \\
\hline Es un desafío profesional distinto dentro de mi trayectoria laboral & 20 \\
\hline Mi vocación de trabajo con estudiantes vulnerables & 19 \\
\hline Mi positiva evaluación del nuevo proceso de selección de directores & 3 \\
\hline La estabilidad laboral del cargo & 0 \\
\hline La remuneración del cargo & 0 \\
\hline Otra (la identificación con mi establecimiento) & 1 \\
\hline Total (\%) & 100 \\
\hline Total (N) & 83 \\
\hline
\end{tabular}

Fuente: Elaboración propia a partir de encuesta directores(as) novatos(as)

\section{b. Una abrupta llegada al establecimiento}

Con posterioridad a su participación exitosa en el proceso de selección, los directores principiantes suelen vivir una dificultosa experiencia de aterrizaje en el establecimiento escolar.

Este shock de realidad se corresponde con la carencia de procesos de inducción al momento de ascender a la posición directiva. Los resultados de la encuesta muestran esta realidad: el 81\% de los novatos declara no haber participado de ningún proceso de inducción. Cuando existe, esta suele consistir en una reunión de carácter protocolar, en que se le brinda al nuevo director información general acerca del establecimiento o sobre las directrices de la educación comunal por parte de funcionarios del municipio.

Otros elementos que suelen dificultar los procesos iniciales de inserción son los siguientes:

- Baja disposición de información previa de los establecimientos: los nuevos directivos disponen frecuentemente solo de información genérica respecto de los establecimientos que dirigirán.

- Inserción en momentos avanzados del calendario escolar: los directores se incorporan al establecimiento en momentos diversos del año escolar, no existiendo una planificación que les permita introducirse en períodos más propicios para conocer la realidad 
existente y realizar una programación de su gestión. La situación más difícil se produce cuando arriban ya avanzado el año escolar.

- Escaso tiempo entre el nombramiento y la asunción del cargo: los directores suelen ser notificados de su nombramiento pocos días o semanas antes de tener que asumir el cargo, lo que impide que dispongan de un tiempo suficiente para reflexionar sobre la nueva tarea que emprenden y de afinar el plan que deberán implementar.

- Falta de entrega formal de la administración del director saliente: no se suele disponer de un proceso formalizado y protocolizado de entrega de la administración -y su respectiva documentación- de parte del director que abandona el establecimiento respecto del que asume la labor.

- Desinformación de las comunidades escolares del nombramiento: hasta su llegada a los establecimientos, es usual que las comunidades educativas no sean informadas por canales formales acerca de la nominación del nuevo director. Esto da lugar a la circulación de rumores y equívocos que deben ser despejados una vez llegado el principiante.

- Mantención de los directores salientes al interior de los establecimientos: no es inhabitual (27\% de los encuestados lo constata) que la administración municipal mantenga en otro rol, por razones administrativas o económicas, al antiguo director dentro de la misma escuela o liceo, lo que genera confusión de roles e incomodidad para la nueva gestión.

Según pudo establecerse en los estudios de caso, la actitud inicial de la comunidad escolar hacia los directores principiantes es variable. Ella oscila, entre otros factores, de acuerdo con la relación que aquella sostenía con el director saliente, así como respecto de las características que posee el entrante. En este sentido, cuando el director saliente tenía un fuerte aprecio y ascendiente entre los directivos, docentes y apoderados, la llegada del nuevo director levanta mayores aprehensiones. Lo mismo ocurre al revés, cuando los directores que son reemplazados mantenían un vínculo conflictivo con la comunidad escolar. De igual manera, hay ciertos rasgos de los propios directores principiantes que pueden concitar un mayor recelo 
por parte de las comunidades escolares, como son llegar desde un establecimiento privado, venir de fuera de la comuna o de la ciudad y ser jóvenes, mientras que otras características pueden llamar a la confianza y a la predisposición positiva: haber desarrollado su carrera profesional en un establecimiento público prestigioso.

Por cierto, la descripción realizada de esta abrupta llegada al establecimiento no es aplicable al grupo de directores insiders, que ya estaban anteriormente trabajando en el centro escolar y que, por ende, tienen un amplio conocimiento previo de su realidad. Sin embargo, estos directivos también enfrentan, como veremos posteriormente, peculiares dificultades al momento de asumir sus nuevas funciones.

\section{c. Prioridades, logros y dificultades del período inicial}

A medida que se integran al establecimiento, los directores noveles van definiendo (discursiva y/o prácticamente) las prioridades de su gestión. Muchas veces, esto implica un reacomodo de sus expectativas iniciales y la focalización en prioridades de corto plazo. Así durante el primer año se busca, antes que todo, resolver las necesidades más críticas y urgentes que presentan los establecimientos, tales como reposicionar la imagen de los centros educativos ante la comunidad, dotarlos de un sello característico, normalizar su funcionamiento (especialmente en lo referido a la asistencia y al trabajo escolar de docentes y alumnos), mejorar el precario equipamiento e infraestructura física, o alcanzar un clima escolar (y de disciplina) positivo. Estas prioridades inmediatas no suelen incluir lo referido a lo técnico-pedagógico, que es visualizado (si acaso) como un ámbito que solo podrá ser abordado posteriormente, cuando se hayan generado ciertas condiciones previas que lo permitirían.

La sistematización de las principales actividades realizadas en los distintos casos estudiados (Cuadro 3) permite dimensionar el amplio despliegue desarrollado por los directores principiantes. 
Cuadro 3. Sistematización de acciones según ámbito de la gestión

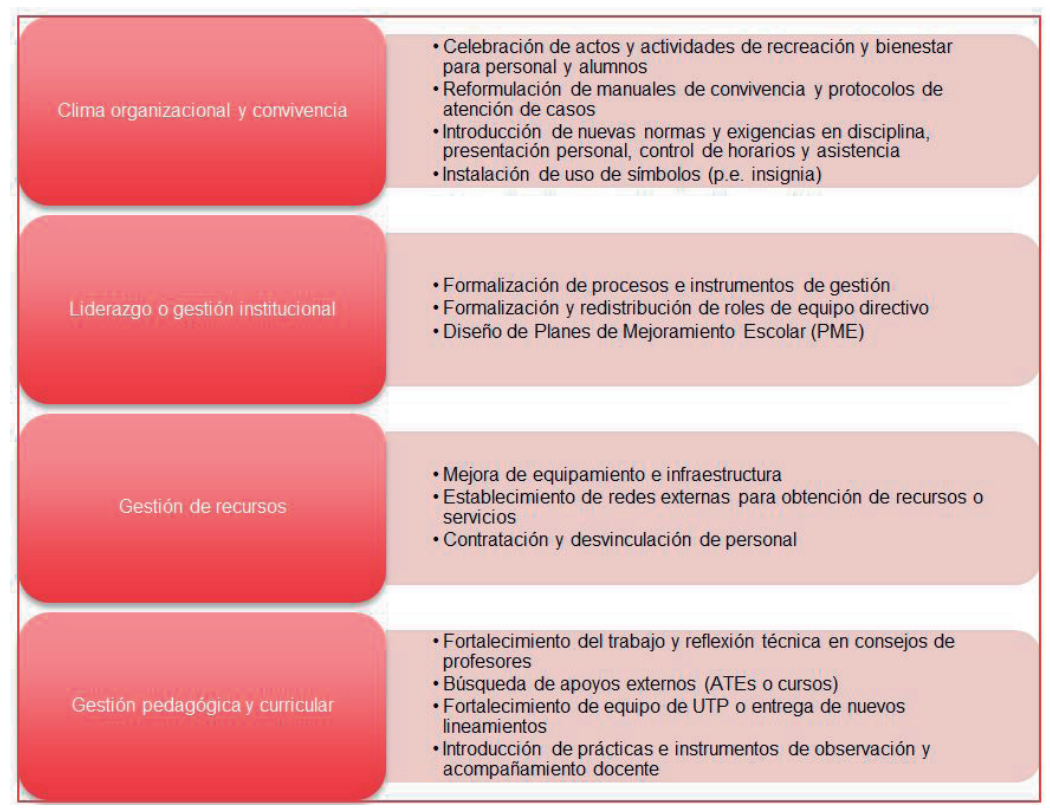

Fuente: Elaboración propia a partir de estudios de caso

La frecuencia de las distintas actividades realizadas y la percepción de logro derivada de ellas fueron motivo de consulta en la encuesta realizada a los directores noveles. El resultado del análisis efectuado ${ }^{8}$ evidenció que las actividades más frecuentes se producen en el ámbito de la gestión del clima y la convivencia, que es también aquel en el que se percibe un mayor avance. Por el contrario, lo referido a la gestión curricular es donde menos actividades se ponen en marcha y donde también se perciben los menores logros. En una situación intermedia, como muestra el gráfico siguiente, se ubican las dimensiones de liderazgo (o gestión) institucional y de gestión de recursos, donde tanto las actividades como sobretodo los logros están a medio camino entre el clima escolar y lo curricular-pedagógico.

8 La encuesta solicitó a los directores que indicaran, en una pregunta abierta y sin orden de importancia, las tres acciones principales que habían desarrollado en sus establecimientos durante su primer año en el cargo, así como los tres logros primordiales de su gestión. En ambos casos las respuestas fueron categorizadas sobre la base de las cuatro áreas de la gestión escolar consideradas por el Marco de la Buena Dirección (Ministerio de Educación, 2005). 
Gráfico 1. Acciones y logros en el primer año en el cargo (\% que menciona al menos 1 acción o logro en cada área de la gestión) ( $N=79$ )

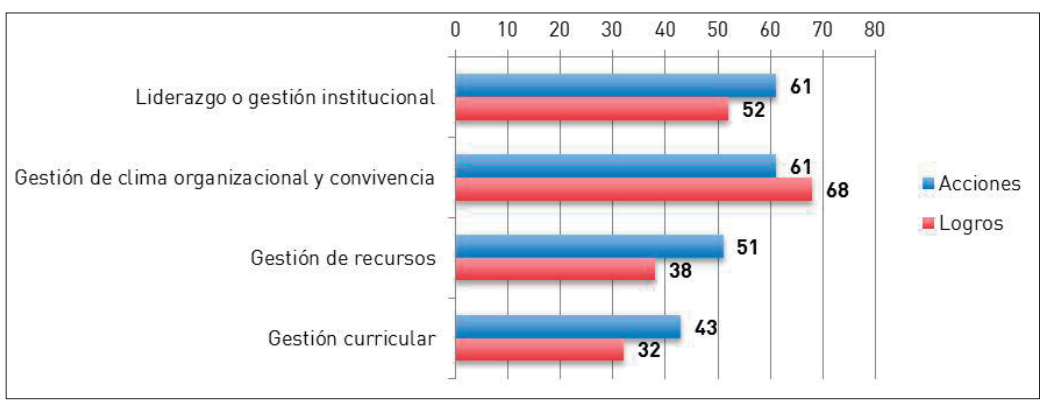

Fuente: Elaboración propia a partir de encuesta directores(as) novatos(as)

Si bien los directores novatos identifican algunos logros en el período en que han encabezado sus respectivos establecimientos escolares, no hay duda de que han vivido una experiencia dificultosa y, en algunos casos, traumática. De hecho, $81 \%$ de los encuestados califica su experiencia como "difícil" o "muy difícil". Las causas son diversas, pero es posible clasificarlas en tres grandes categorías: las externas al establecimiento (restricciones que se imponen a la gestión por parte de la administración municipal o las políticas educativas), las internas al establecimiento (propias de la dinámica que acontece en el funcionamiento de la comunidad escolar) y las personales (derivadas de las limitaciones en las propias competencias y conocimientos de los directores) (Cuadro 4). Si bien esta clasificación permite ordenar las dificultades, debe considerarse que en la experiencia de los propios directivos ellas aparecen muchas veces conectadas entre sí (p. ej., la falta de atribuciones directivas respecto del manejo de los recursos humanos dentro del establecimiento escolar hace que ellos no puedan hacer frente a la falta de profesionalismo de ciertos docentes). Adicionalmente, debe consignarse que la experiencia de esta cohorte 2014-2015 de directores principiantes está marcada por un acontecimiento particular y de muy complejo manejo para la dirección del establecimiento: el desarrollo de una larga paralización de actividades del profesorado, de casi dos meses, convocada por el Colegio de Profesores y seguida mayoritariamente por los establecimientos municipales, producto de su rechazo a la nueva Ley de Carrera Docente presentada por el Gobierno al Congreso. 
Cuadro 4. Sistematización de dificultades centrales enfrentadas por área

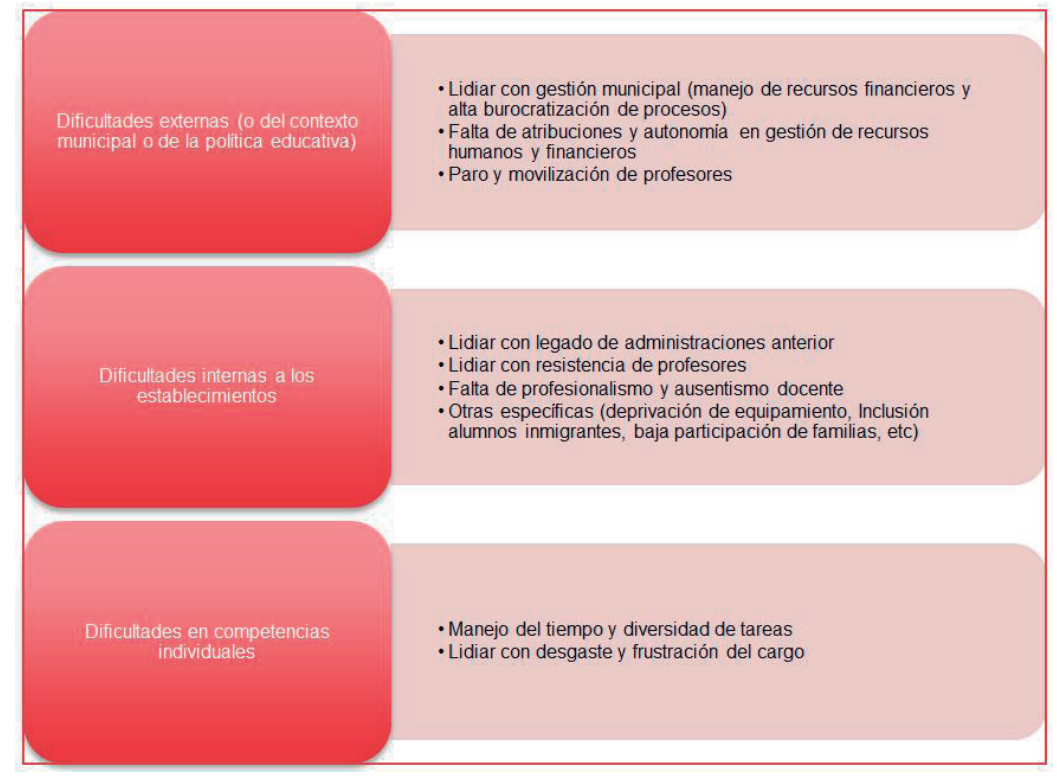

Fuente: Elaboración propia a partir de estudios de casos y encuesta directores(as) novatos(as)

Si bien esta panorámica de dificultades resulta común a la mayoría de los directores principiantes, hay ciertos matices que conviene subrayar. Así los directivos que no provienen del sector público resienten mucho más la burocracia de la administración municipal y la politización atribuida a algunos de sus nombramientos, así como tienen un repertorio de respuestas mucho más limitado frente a coyunturas de movilización sociopolítica, como la ya mencionada paralización de actividades docentes ${ }^{9}$. Igualmente los directores insiders deben afrontar la ardua tarea de validar su liderazgo y autoridad frente a sus antiguos pares, enfrentando ciertos conflictos inevitables de roles e identidad profesional y debiendo maniobrar adecuadamente (y con ecuanimidad) frente a las disputas,

9 Los casos observados dieron cuenta de cómo la paralización de actividades en un establecimiento municipal pone en una situación de alta complejidad a sus directivos que deben gestionar difíciles equilibrios entre los intereses de distintos actores (docentes, apoderados, etc.). En este sentido, los directores deben velar por que la movilización no signifique un quiebre dentro del mismo profesorado de la escuela o liceo, así como que no traiga perjuicios mayores a su funcionamiento (p. ej. fuga de apoderados y perdida de matrícula o retroceso irremontable en la cobertura curricular prevista). 
afinidades y rivalidades profesionales y personales que precedían su nombramiento.

d. La primacía de la propia experiencia directiva y de los apoyos informales

A pesar de las dificultades vividas en su primer período a cargo del establecimiento, los directores principiantes no se cuestionan su idoneidad profesional y creen de forma mayoritaria que estaban bastante o muy preparados para asumir esta responsabilidad.

Para indagar más respecto de dicha preparación, en la encuesta se les consultó por las dos experiencias de su trayectoria formativa y laboral que les habían significado un mayor aporte a su aprendizaje para el cargo. Así ellos otorgan, en primer lugar, una alta valoración al "ejercicio previo de cargos directivos" (58\%), en segundo lugar a "la participación en instancias especializadas de formación para el cargo" (52\%), y, más abajo, consideran a "la observación del desempeño de los directores escolares de los establecimientos en que se trabajó con anterioridad" (33\%). De manera que, recuperando los conceptos enunciados anteriormente, se produce una combinación entre elementos que ellos han adquirido en la socialización preparatoria con otros que provienen de su socialización profesional.

Una vez investidos en el cargo, los directores noveles plantean haber recibido un apoyo relevante a su gestión de parte de actores internos a los centros educativos. Al solicitarles que evaluaran la importancia de los diferentes apoyos internos, los directivos destacaron el soporte brindado, en orden decreciente, por los estudiantes, la secretaria, el personal no docente y los directivos y docentes del establecimiento -todos los que reciben un alto porcentaje de respuesta afirmativa de entre tres cuartos (3/4) y dos tercios (2/3) de los encuestados-. Un escalón más abajo, siendo vistos como un respaldo por parte de algo más de la mitad de los principiantes, se ubican los apoderados y los dirigentes gremiales.

La misma consulta, pero formulada respecto de los apoyos externos, muestra que el primer círculo de apoyo está constituido por 
otros colegas directores que forman parte de las redes personales de cada uno de los novatos. Los estudios de caso corroboraron la alta importancia asignada a estas redes informales en desmedro de los soportes institucionales.

La figura del "director(a) amigo(a)" aparece como un mecanismo clave para la resolución y consulta de inquietudes de la gestión cotidiana, para brindar soporte en temas técnicos o administrativos, o para la entrega de consejos y sugerencias de acción frente a conflictos con actores internos y/o externos al establecimiento. Se confía en la utilidad de la experiencia, el conocimiento acumulado y el buen criterio de este directivo cercano, pero también en su discreción respecto del uso de la información entregada y en su auténtico interés en el éxito de la gestión en curso.

Los apoyos institucionales tienen un lugar más secundario. Con todo, el más cercano es el sostenedor, mientras que hay una relación mucho más distante con los funcionarios del Ministerio de Educación o de la Agencia de la Calidad. Las instituciones privadas de asistencia técnica (ATES) prácticamente no son mencionadas como apoyo. Empero, como ya vimos, esta relación con el sostenedor municipal es cuestionada por los principiantes por su alta burocratización, considerándose que se les realizan muchas demandas de tipo administrativo, así como muy frecuentes solicitudes de asistencia a actividades de distinto tipo que los distraen de su labor central en los establecimientos. De hecho, y en conexión con la falta de inducción ya reseñada, solo el $27 \%$ de los directivos encuestados estuvo de acuerdo con la afirmación de que "el sostenedor y su equipo han realizado un acompañamiento y retroalimentación de carácter técnica permanente a mi gestión en el primer año en el cargo". Esta visión crítica de los sostenedores se agudiza entre quienes no provienen del sector municipal, incorporando a veces nuevas temáticas tales como la politización atribuida a ciertos nombramientos dentro del establecimiento o la dificultad de modificar el equipo directivo. Conviene consignar que si bien el Ministerio de Educación y la Agencia de la Calidad son vistos como actores distantes, las orientaciones generales que ellos han entregado para el ejercicio de la dirección escolar (Marco para la Buena Dirección, Estándares Indicativos de 
Desempeño) sí son valoradas como herramientas útiles por parte de los directores noveles.

\section{Conclusiones y discusión}

Al inicio de este artículo se planteó el escaso conocimiento del que se dispone en Chile acerca de los directores principiantes de los establecimientos municipales, a pesar de que su número -dadas las obligaciones emanadas de la Ley $N^{\circ} 20.501$ - es creciente y de que su desempeño es estratégico para la mejora escolar. A continuación, se desea recapitular los principales hallazgos del estudio realizado, para luego plantear algunas discusiones relevantes que emanan de ellos.

Un primer hallazgo radica en que es posible elaborar un perfil con las características más destacadas de los principiantes que, en la actualidad, están asumiendo la posición directiva. Dicho perfil puede apreciarse sintéticamente en la Tabla 2.

Tabla 2. Perfil de los directores novatos que ingresaron al sector municipal en años 2014-2015

\begin{tabular}{ll}
\hline 2/3 & Son mujeres \\
\hline $4 / 5$ & poseen un grado de magister \\
\hline 20 & años de experiencia previa en promedio (con alta dispersión) \\
\hline $1 / 2$ & ha sido previamente Jefe de Unidad Técnico-Pedagógica \\
\hline $4 / 5$ & ha ejercido previamente algún cargo directivo \\
\hline $3 / 4$ & ha trabajado previamente en establecimientos privados \\
\hline $4 / 5$ & llega al cargo desde un establecimiento escolar diferente al que trabajaba (outsider) \\
\hline
\end{tabular}

Este perfil es parcialmente distinto al de los directores que hoy de forma mayoritaria ejercen en la dependencia municipal, fundamentalmente en torno a una mayor presencia femenina y la preparación especializada (grado de magíster).

El perfil profesional de los novatos puede complementarse con un rasgo más subjetivo, referido a su motivación para asumir esta posición. Es así como existe una contundente mayoría que declara arribar al rol de dirección con una motivación de orientación 
pública, como apoyar la gestión de la educación municipal o trabajar con alumnos vulnerables. En contrapartida, no se está atraído por la estabilidad (de hecho la ley obliga a una renovación vía concurso cada cinco años y no permite más de dos períodos sucesivos en el mismo establecimiento) ni por la renta (la que es vista derechamente como insuficiente por los nuevos directores).

Un segundo hallazgo refiere a la abrupta y dificultosa forma en que los directores novatos suelen asumir sus funciones en los establecimientos. En efecto, las condiciones en que ellos asumen sus nuevas labores no suelen brindarles ciertas facilidades básicas para instalarse en el rol y más bien tienden a tensionarles tanto a ellos mismos como a las comunidades escolares que empiezan a comandar. Factores tales como la ausencia de una entrega formal del estado de situación educativo y administrativo del establecimiento por parte de la dirección saliente, la instalación realizada en cualquier período del año escolar, la precaria información hacia la comunidad escolar del proceso de reclutamiento realizado o el escaso tiempo de preparación entre el nombramiento del principiante y su asunción del cargo apuntan en esta dirección.

Adicionalmente el estudio constata que la gran mayoría de los directores noveles (cuatro de cada cinco) no cuenta con un proceso de inducción en el cargo y en la organización. Y entre aquellos que sí tienen alguna inducción, esta tiende a ser breve, formal y solo informativa de las políticas del sostenedor municipal.

Un tercer hallazgo describe las actividades prioritarias que, una vez instalados, realizan los novatos en la dirección de sus establecimientos. Si bien se aprecia que intentan desarrollar muchas actividades y de muy distinto tipo, es claro que las referidas a la gestión pedagógica y curricular son las menos frecuentes. Predominan, en cambio, las actividades relacionadas con el clima y la convivencia dentro del establecimiento, así como aquellas dirigidas a la gestión institucional. Pese a que la definición de estas primeras acciones surge muchas veces como producto de la propia motivación de los novatos, el estudio revela que tanto las exigencias del exterior (Ministerio, sostenedor municipal) como las características particulares de la 
escuela son también determinantes a la hora de establecer el curso de acción en el primer año. Los hallazgos de este estudio muestran que los novatos buscan adecuar su accionar al nivel de desempeño de la escuela, siendo frecuente que concentren su tiempo en tareas asociadas a la normalización de la gestión educativa de la misma.

Esta identificación de acciones más y menos frecuentes se complementa con la opinión de los principiantes respecto de los logros que consideran que han alcanzado en este período inicial de dirección. Así, en línea con su nivel de actividad, los directores noveles consideran que donde más han logrado avanzar es en materias de clima y convivencia escolar, mientras que en los aspectos relacionados con lo pedagógico y curricular son los que menos avances obtienen.

Un cuarto hallazgo refiere a que la experiencia es vivida por la gran mayoría los novatos como difícil o muy difícil. Este juicio evaluativo se basa en la convergencia hacia ellos de múltiples presiones, que provienen desde diferentes actores y no están planificadas en el tiempo, ante las cuales deben ensayar respuestas que involucran mucha dedicación y que, aun así, muchas veces no logran ser satisfactorias. Los requerimientos administrativos que provienen desde fuera del establecimiento escolar (sostenedor municipal, Ministerio de Educación, Agencia de Calidad, Superintendencia de Educación) les implican un despliegue significativo. Pero además la resolución de temas internos del establecimiento exige la intervención de estos agentes externos, en particular del sostenedor municipal, y los directivos no poseen atribuciones ni herramientas para movilizar dichos recursos institucionales ni para encuadrar estos procesos de toma de decisión en los plazos requeridos (p. ej., el reemplazo de docentes con licencia médica). Adicionalmente deben considerarse los problemas propios de la dinámica escolar que suelen acontecer dentro de un establecimiento municipal, en que el ausentismo de alumnos y docentes, los problemas de infraestructura, la baja preparación profesional de algunos docentes o el descompromiso de las familias con la educación son recurrentes. Si bien la evidencia nacional releva que estas dificultades internas y externas son generalizables más allá de los novatos (Weinstein y Muñoz, 2012), estos últimos tienen 
menor experiencia, redes de contacto y competencias profesionales para hacerles frente que quienes ya poseen cierta trayectoria en la dirección escolar.

Un último hallazgo refiere a los recursos personales y apoyos con que, según su propio punto de vista, cuentan los principiantes para enfrentar su período de inicio en el cargo. En este sentido, los novatos valoran especialmente ciertos aprendizajes que hicieron previamente a la asunción del cargo, como son el paso por distintos roles directivos y la realización de estudios especializados para la función de dirección. Igualmente ellos consideran que las comunidades escolares en las que se han insertado, partiendo por sus alumnos, les han apoyado en su tarea. Son más críticos, en cambio, respecto de los apoyos institucionales externos que han recibido. Y hacen visible la existencia de una red de apoyo informal entre pares, constituida por otros directores con que guardan vínculos afectivos y de amistad, los que se constituyen en un referente decisivo a la hora de resolver problemas y tomar decisiones.

Los resultados de este estudio pueden ser analizados en relación con la literatura especializada sobre los directores novatos, así como respecto de requerimientos específicos de las políticas favorables al liderazgo directivo escolar en Chile. Al respecto interesa focalizarse brevemente en cuatro temas.

Un primer tema se relaciona con la heterogeneidad existente entre los principiantes, tanto en lo referido a su socialización como en relación con su experiencia en el cargo. En efecto, más allá de los elementos comunes y compartidos, existen algunas características específicas de los procesos de socialización anticipatoria de los directores novatos chilenos que inciden en su experiencia de ejercicio del cargo. Profundizado en un tópico escasamente abordado por la literatura internacional y que presenta controversia en sus resultados (Daresh \& Male, 2000; Hobson et al., 2003, Parway \& Hall, 1992; Spillane \& Lee, 2013), la investigación efectuada establece que ciertos elementos particulares de las trayectorias laborales de los nuevos directores ejercen una influencia importante en el tipo de conocimientos y habilidades con que ascienden al rol y, 
concomitantemente, en los déficits formativos que poseen. Dentro de estos elementos, destacan tres antecedentes clave:

a. Ser insider/outsider: si bien quienes transitan al cargo de director en la misma organización en que se desempeñaban tienen la ventaja de disponer de una familiarización previa con la organización y la comunidad educativa (Spillane \& Lee, 2013), ellos experimentan -incluso por sobre los outsiders- una alta dificultad en el cargo, producto del necesario establecimiento de nuevos límites con sus anteriores pares, sus propios conflictos identitarios de rol y la compleja validación de su liderazgo.

b. La dependencia de origen: los novatos que provienen del sector particular y transitan por primera vez a un establecimiento público se enfrentan a dificultades específicas que emanan de su desconocimiento de la gestión y normativa municipal y de la frecuente mayor resistencia de los docentes a su arribo al cargo.

c. El tipo de cargo directivo desempeñado con anterioridad: aunque exploratoriamente, el estudio evidencia la incidencia del tipo de cargo directivo desempeñado previamente en el estilo de liderazgo ejercido y, en cierta medida, en los focos de las acciones ejecutadas en el primer año, visualizándose una distinción entre haber ejercido el rol de jefe técnico versus otros cargos (como inspector general) en una mayor preocupación por ejercer, aunque sea en el mediano plazo, un liderazgo pedagógico. Las políticas de apoyo respecto de los directores noveles debiesen ser sensibles a estas distinciones.

Un segundo tema relevante refiere a las necesidades formativas detectadas entre los novatos y que debieran formar parte de un eventual proceso de inducción hoy inexistente. Aun cuando los aprendizajes adquiridos en los procesos de socialización profesional -en este caso en la formación de preservicio-son un recurso valorado, ellos no son suficientes para dar respuesta a las complejidades del proceso de inserción en el cargo. En línea con la insuficiencia detectada por la evidencia internacional respecto de la efectividad de los programas de formación de preservicio para el ejercicio del rol por parte de los novatos (Crow, 2007; Daresh \& Male, 2000; Kelly $\&$ Saunders, 2010) y con las específicas debilidades detectadas en 
la oferta formativa a nivel nacional (Muñoz y Marfán, 2012), esta investigación revela que, a pesar de la extensa formación especializada con que cuentan los nuevos directores, esta presenta severos déficits a nivel de los conocimientos profesionales entregados (particularmente en normativa y gestión municipal), al mismo tiempo que carece de métodos de formación experienciales y prácticos capaces de potenciar la transferencia de los contenidos aprendidos para el ejercicio efectivo del rol. En este sentido es relevante identificar las necesidades formativas en sus distintas dimensiones (Clarke \& Wildy, 2013). Esta investigación detecta al menos cuatro grupos de necesidades formativas entre los actuales directores noveles chilenos, a saber:

La entrega de conocimientos profesionales, principalmente en materia de políticas educativas nacionales y locales, que están siendo insuficientemente abordados por el preservicio.

El fortalecimiento de habilidades conductuales y técnicas que resultan clave para enfrentar las tareas centrales asignadas a los directores (como las provenientes del liderazgo pedagógico), así como las dificultades habitualmente presentes en los establecimientos públicos (p. ej., bajo capital cultural de los estudiantes y sus familias).

El apoyo de corte personal y emocional que permita afrontar con éxito las distintas exigencias, presiones y dificultades que presenta el cargo.

El apoyo a la socialización organizacional de cada director en su establecimiento escolar específico, lo que implica una adecuada lectura del contexto y lugar al que arriba, el entendimiento de su cultura organizacional y del sistema en el cual el establecimiento se ubica. Si pretenden ser pertinentes, los procesos de inducción deben considerar las distintas situaciones que enfrentan los principiantes. En efecto, aun cuando las necesidades formativas y de apoyo de los directores muestran ciertos núcleos transversales, ellas son fuertemente contingentes a las particularidades de los centros educativos y a las trayectorias individuales y la socialización previa de los individuos. Por ello, cualquier programa de inducción que se diseñe, debiese contar con la suficiente flexibilidad en sus contenidos y metodologías como 
para responder a las variadas necesidades individuales y de contexto de los principiantes.

Un tercer tema refiere a puntualizar aquellos aspectos problemáticos de la experiencia de los novatos chilenos que difieren de lo habitualmente establecido por la literatura especializada. Así, por una parte, existe un conjunto de desafíos que son comunes a los encontrados en otras latitudes del mundo (García Garduño et al., 2011 ; Oplatka, 2012; Spillane \& Lee, 2013) y que demuestran, tal como la literatura establece, ser relativamente independientes del sistema escolar del cual se trate (Nelson \& Slater, 2013). Dentro de estos, se ubican aquellos de carácter interno a los centros educacionales, como el manejo del legado de los directores anteriores, el lidiar con el cuerpo docente y sus problemáticas, la necesidad de reacomodar las expectativas iniciales en la gestión o bien la ausencia de competencias de los principiantes para afrontar la complejidad del ejercicio del rol -en especial la diversidad y amplitud de tareas enfrentadas por la dirección escolar-. Pero, por otra parte, el estudio enfatiza la existencia de problemáticas de carácter externo o de la política educativa del sistema escolar chileno que ejercen una influencia crítica en la experiencia de desempeño del cargo directivo. Así, tal como da cuenta una investigación nacional de directores escolares en servicio (Ulloa et al., 2012), este estudio detecta dificultades en los novatos para gestionar procesos clave del funcionamiento del establecimiento que son producto de la burocracia de las autoridades municipales y la falta de atribuciones en materia de recursos humanos y financieros (al mismo tiempo que se le exige y responsabiliza por el cumplimiento de exigentes metas académicas y de matrícula escolar). Además, los resultados dejan al descubierto las tensiones al interior de las comunidades a causa de la movilización sociopolítica nacional -que llegó al límite con el paro de profesores acontecido en el transcurso de la investigación realizada-. En este sentido, la investigación confirma la alta incidencia del contexto socioeducativo en la experiencia en el primer año de los nuevos directivos (Weinstein y Hernández, 2014).

Un último tema refiere a la carrera directiva y a cómo la etapa de inicio, que corresponde al director novato, puede verse alterada dependiendo de su definición y de los prerrequisitos establecidos para 
acceder a ella. La literatura especializada plantea que antes de arribar al cargo, los directores han pasado por procesos de socialización continuos e incrementales (Duke, 1987), que los han provisto de ciertos recursos básicos a nivel de conocimientos, habilidades y principios para el ejercicio del rol, y los han empoderado para asumir el desafío profesional que este encierra. La evidencia aportada por esta investigación establece que, aun cuando la política educativa del país carece de una carrera directiva formalmente instituida y, por ende, no existe una trayectoria común preestablecida a seguir (p.ej., ni el paso por cargos directivos ni la aprobación de ciertos programas formativos son obligatorios), los nuevos directores destacan por la realización de trayectorias laborales y formativas semejantes, las que, a su vez, organizan sus procesos de socialización anticipatoria y profesional. En coherencia con la literatura internacional, aparecen así como principales fuentes de aprendizaje el ejercicio previo de cargos directivos y la disposición de formación especializada, específicamente magísteres en gestión o liderazgo escolar. De modo que los aprendizajes previos para el rol del cual disponen los principiantes al ascender al cargo se ejecutan por medio de una combinación de tácticas de socialización (Bengston, Zepeda \& Parylo, 2013; García Garduño et al., 2011a), tanto secuenciales, como formales e informales, y colectivas e individuales, las cuales los han provisto de un cúmulo de conocimientos técnicos, conductuales y valóricos, que son puestos al servicio del ejercicio del cargo en el primer año. La construcción de una eventual carrera directiva en el sistema escolar chileno debiera partir por reconocer la existencia de facto de estos escalones previos en la experiencia y la socialización mayoritaria de los directores novatos para avanzar hacia un sistema más organizado, equitativo y confiable, que dependa menos de los vaivenes de la formación previa y de las redes personales de apoyo de cada principiante. En esta nueva carrera directiva debieran garantizarse procesos de inducción y de apoyo flexibles y de calidad para los directores principiantes, de manera que no solo su experiencia profesional (y personal) sea menos dificultosa, sino que se potencie su liderazgo educativo y su incidencia en la mejora escolar de las comunidades escolares que encabezan. 


\section{Referencias}

Ahumada, L. (2012). Liderazgo en organizaciones educativas. Persona, 2012(15), 239-252.

Bengtson, E., Zepeda, S. J., \& Parylo, O. (2013). School systems' practices of controlling socialization during principal succession looking through the lens of an organizational socialization theory. Educational Management Administration E Leadership, 41(2), 143-164. http://dx.doi. org/10.1177/1741143212468344

Blase, J. J. (1985). The socialization of teachers an ethnographic study of factors contributing to the rationalization of the teacher $>$ instructional perspective. Urban Education, 20(3), 235-256. http://dx.doi. org/10.1177/004208598502000301

Blase, J. J. (1986). Socialization as humanization: One side of becoming a teacher. Sociology of Education, 59(2), 100-113. http://dx.doi. $\operatorname{org} / 10.2307 / 2112435$

Bush, T. (2012). International perspectives on leadership development: making a difference. Professional Development in Education, 38(4), 663678. http://dx.doi.org/10.1080/19415257.2012.660701

Clarke, S. \& Wildy, H (2013). Investigating preparation for the principalship: deliberating on possibilities. En C. Slater \& S. Nelson, S. (Eds.), Understanding the principalship: an international guide to principal preparation (pp. 25-44). Bradford: Emerald Group Publishing Limited.

Cohen, L., Manion, L., \& Morrison, K. (2011). Research methods in education. Abingdon, New York: Routledge.

Crow, G. M. (2007). The professional and organizational socialization of new English headteachers in school reform contexts. Educational Management Administration E Leadership, 35(1), 51-71. http://dx.doi. org/10.1177/1741143207071385

Daresh, J. (abril, 1987). The highest hurdles for the first year principal. Trabajo presentado en Annual Meeting of the American Educational Research Association, Washington DC.

Daresh, J. \& Male, T. (2000). Crossing the border into leadership: experiences of newly appointed British headteachers and American principals. Educational management administration \& leadership, 28(1), 89-101. http://dx.doi.org/10.1177/0263211x000281013

Day, C., Hopkins, D., Harris, A., \& Ahtaridou, E. (2009). The impact of school leadership on pupil outcomes (Reporte final). Nottingham: University of Nottingham. 
42 DIRECTOR(A) POR PRIMERA VEZ. UN ESTUDIO SOBRE LA EXPERIENCIA Y SOCIALIZACIÓN DE LOS DIRECTORES NOVELES EN ESTABLECIMIENTOS MUNICIPALES DE CHILE - J. Weinstein, C. Cuéllar, M. Hernández y M. Fernández

Duke, D. (1987). School leadership and instructional improvement. New York: Random House.

Earley, P. (2012). Observation methods: learning about leadership practice through shadowing. Journal of Educational, Cultural and Psychological Studies, 3(6), 15-31. http://dx.doi.org/10.7358/ecps-2012-006-earl

Earley, P., Nelson, R., Higham, R., Bubb, S, Porritt, V., \& Coates, M. (2011). Experiences of new headteachers in cities (Reporte de investigación). Nottingham: National College for School Leadership.

García Garduño, J.M., Slater, C. y López-Gorosave, G. (2011a). El director escolar novel: estado de la investigación y enfoques teóricos. Revista Iberoamericana sobre Calidad, Eficacia y Cambio en Educación, 9(3), 31-50.

García Garduño, J.M., Slater, C., \& López-Gorosave, G. (2011b). Beginning elementary principals around the world. Management in Education, 25(3), 100-105. http://dx.doi.org/10.1177/0892020611403806

Greenfield, W. (April, 1985). Being and becoming a principal: responses, contexts and socialization processes. Trabajo presentado en Annual Meeting of American Educational Research Association, Chicago, Illinois.

Greenfield, W. Jr. (1977). Administrative candidacy: A process of new-role learning, part I. Journal of Educational Administration, 15(1), 30-48. http://dx.doi.org/10.1108/eb009763

Grupo Educativo (2015) Evaluación de la implementación del sistema de selección de directores en el marco de la Ley $N^{o} 20.501$ (Documento no publicado). Santiago.

Hallinger, P., \& Huber, S. (2012). School leadership that makes a difference: international perspectives. School Effectiveness and School Improvement, 23(4), 359-367. http://dx.doi.org/10.1080/09243453.2012.681508

Hargreaves, A., Moore, S., Fink, D., Brayman, C., \& White, R. (2003). Succeeding leaders? A study of principal succession and sustainability (Reporte de investigación). Toronto: Ontario Principals Council.

Hobson, A., Brown, E., Ashby, P., Keys, W., Sharp, C, \& Benefield, P. (2003). Issues for early headship - problems and support strategies (Reporte de investigación). Nottingham: National College for School Leadership.

Kelly, A. \& Saunders, N. (2010). New head son the block: three cases studies of transition to primary school headship. School Leadership \& Management, 30(2), 127-142. http://dx.doi.org/10.1080/13632431003663180

Lacey, C. (1977). The socialization of teachers. London: Methuen. 
LeCompte, M. \& Preissle, J. (1993). Ethnography and qualitative design in educational research. London: Academic Press.

Leithwood, K., Steinbach, R., \& Begley, P. (1992). Socialization experiences: becoming a principal in Canada. En F.W. Parkay, y G.E. Hall (Eds.), Becoming a principal the challenges of beginning leadership (pp. 284-307). Needham Heights, MA: Allyn \& Bacon.

McDonald, S. (2005). Studying actions in context: A qualitative shadowing method for organizational research. Qualitative Research, 5(4), 455-473. http://dx.doi.org/10.1177/1468794105056923

Ministerio de Educación, Mineduc (2005). Marco para la buena dirección, criterios para el desarrollo profesional y evaluación de desempeño. Recuperado de http://www.mineduc.cl/usuarios/convivencia_escolar/ doc/201103070155490.MINEDUC.Marco_para_la_Buena_Direccion. pdf

Ministerio de Educación, Mineduc (2015). Aprueba bases administrativas y técnicas, y anexos, para el concurso de proyectos sobre la creación de centros de liderazgo escolar. Recuperado de http://mineduc.cl/ usuarios/mineduc/File/2015/BASES\%20CENTROS\%20DE\%20 LIDERAZGO\%20ESCOLAR\%20TOTALMENTE\%20TRAMITADAS. pdf

Muñoz, G. y Marfán, J. (2012). Formación de directores escolares en Chile: características y desafíos. En J. Weinstein y G. Muñoz (Eds.) ¿Qué sabemos sobre los directores de escuelas en Chile? (pp. 83-110). Santiago de Chile: Fundación Chile y Pontificia Universidad Católica de Chile.

Nelson, S., \& Slater, C. (2013). Lessons in leadership from the vineyard. En C. Slater \& S. Nelson (Eds.), Understanding the principalship: an international guide to principal preparation (pp. 3-24). Bradford: Emerald Group Publishing Limited

Oplatka, I. (2012). Towards a conceptualization of the early career stage of principalship: current research, idiosyncrasies and future directions. International journal of leadership in education: theory and practice, 15(2), 129-151. http://dx.doi.org/10.1080/13603124.2011.640943

Parkay, F.W., \& Hall, G.E. (1992). Becoming a principal: the challenges of beginning leadership. Needham Heights, Massachusetts: Allyn and Bacon.

Rodríguez, S.; Cárdenas, F. y Campos, F. (2012). El desarrollo del liderazgo educativo de un estudio de historias de vida. Revista Iberoamericana sobre Calidad, Eficacia y Cambio en Educación, 10(1), 1-9.

Shenton, A. K. (2004). Strategies for ensuring trustworthiness in qualitative research projects. Education for Information, 22(2), 63-75. 
44 DIRECTOR(A) POR PRIMERA VEZ. UN ESTUDIO SOBRE LA EXPERIENCIA Y SOCIALIZACIÓN DE LOS DIRECTORES NOVELES EN ESTABLECIMIENTOS MUNICIPALES DE CHILE - J. Weinstein, C. Cuéllar, M. Hernández y M. Fernández

Spillane, J. \& Lee, L. (2013). Novice school principals' sense of ultimate responsibility: problems of practice in transitioning to the principal's office. Educational Administration Quarterly, 50(3), 431-465. http:// dx.doi.org/10.1177/0013161x13505290

Teddlie, C. \& Tashakkori, A. (2006). A general typology of research design featuring mixed methods. Research in the Schools, 13(1), 12-28.

Ulloa, J.; Nail, O.; Castro, A. y Muñoz, M. (2012). Problemas de gestión asociados al liderazgo como función directiva. Estudios Pedagógicos, 38(1), 121129. http://dx.doi.org/10.4067/s0718-07052012000100007

Universidad Diego Portales, UDP (2014). Encuesta La voz de los directores 2013. (Documento no publicado). Santiago de Chile.

Van Maanen, J., \& Schein, E.H. (1979). Towards a theory of organizational socialization. Research in Organizational Behavior. Recuperado de http:// dspace.mit.edu/bitstream/handle/1721.1/1934/SWP-0960-03581864. pdf

Walker, A. \& Qian, H. (2006). Beginning principals: balancing at the top of the greasy pole. Journal of Educational Administration, 44(4), 297-309. http://dx.doi.org/10.1108/09578230610674921

Weindling, D. (abril, 2000). Stages of headship: a longitudinal study of the principalship. Trabajo presentado en The Annual Meeting of the American Educational Research Association. New Orleans, LA.

Weindling, D. \& Earley, P. (1987) Secondary headship: the first years. Windsor: NFER-Nelson

Weinstein, J. y Hernández (2014). Mentoría y redes entre directores de escuela: evidencia y orientaciones de política para el fortalecimiento del aprendizaje entre pares. (Documento no publicado elaborado para OECD). Santiago de Chile.

Weinstein, J. \& Hernández, M. (2015a). Birth pains: emerging school leadership policies in eight school system of Latin America. International Journal of Leadership in Education: Theory and Practice, 19(3), 241-263. http://dx.doi.org/10.1080/13603124.2015.1020344

Weinstein, J. y Hernández, M. (2015b). ¿Un centro nacional de liderazgo escolar en Chile? Antecedentes para su diseño. Recuperado de: http:// www.espaciopublico.cl/media/publicaciones/archivos/44.pdf

Weinstein, J y Muñoz, G. (2012) ¿Qué sabemos sobre los directores de escuelas en Chile? Santiago de Chile: Fundación Chile y Pontificia Universidad Católica de Chile. 
Weinstein, J., Muñoz, G., \& Raczynski, D. (2011). School leadership in Chile: Breaking the inertia. En T. Townsend, T. y J. McBeath (Eds.), International handbook of leadership for learning (pp. 297-317). Dordrecht, Heidelberg, London \& New York: Springer.

Wentworth, W.M. (1980). Context and Understanding: An Inquiry into Socialization Theory. New York: Elsevier.

Yin, R. (1999). Research. Design Methods. Fourth Editions, Sage Publications.

Recibido: 29/03/2016

Aceptado: 28/06/2016 